\title{
Effect of California Tri-Pull Taping Method on Shoulder Subluxation, Pain, Active Range of Motion and Upper Limb Functional Recovery After Stroke - A Pretest Post Test Design
}

\author{
Subhasish Chatterjee ${ }^{1, ~ *}$, Narkeesh Arumugam ${ }^{2}$, Divya Midha ${ }^{1}$, Manu Goyal ${ }^{1}$, Ashima Arora ${ }^{1}$, \\ Sorabh Sharma ${ }^{1}$, Senthil P. Kumar ${ }^{1}$
}

${ }^{1}$ Maharishi Markandeshwar Institute of Physiotherapy and Rehabilitation (MMIPR), Mullana - Ambala, Haryana, India

${ }^{2}$ Punjabi University, Patiala, Punjab, India

\section{Email address:}

subhasishphysio@gmail.com (S. Chatterjee),narkeesh@gmail.com (N. Arumugam), divyamidha.pt@gmail.com (D. Midha), manu.goyal1111@gmail.com (M. Goyal), ashima.physio@mmumullana.org (A. Arora), sorabhsharma3@gmail.com (S. Sharma), principal.mmipr@mmumullana.org (S. P. Kumar)

\section{To cite this article:}

Subhasish Chatterjee, Narkeesh Arumugam, Divya Midha, Manu Goyal, Ashima Arora, Sorabh Sharma, Senthil P. Kumar. Effect of California Tri-Pull Taping Method on Shoulder Subluxation, Pain, Active Range of Motion and Upper Limb Functional Recovery After Stroke - A Pretest Post Test Design. American Journal of Psychiatry and Neuroscience. Vol. 3, No. 5, 2015, pp. 98-103. doi: 10.11648/j.ajpn.20150305.14

\begin{abstract}
Objective:The primary objective of the study was to evaluate the effect of California tri-pull taping (CTPT) method on post stroke shoulder subluxation, pain, active range of motion and upper limb functional recovery. Design: Pretest post test design. Setting: Study was conducted ininpatient and outpatient department of MM hospital Mullana- Ambala. Participants: 10 subjects with post stroke shoulder subluxation were included into the study. (7 male , 3 female). Intervention: For taping, two types of tape was used, cotton pre-tape and rigid post-tape. Tape was applied to subjects for thrice a week, for six weeks and conventional neuro rehabilitation programmewas also given to the subjects, five days a week for six weeks. Main outcome measures: Pre, and post assessment scores were taken from each subject by using, Digital Vernier caliper, visual analogue scale (VAS), Goniometer, and Fuglmeyer scale (FUG). Results: The CTPT method produced significant reduction on inferior subluxation from pre intervention to post intervention, pain. There was also significant improvement of AROM, and FUG. Conclusion: This intervention is a promising adjunct to the management of the hemiplegic subluxed shoulder. The main limitation of the study was, small sample size and no control group was used.
\end{abstract}

Keywords: Stroke, Shoulder Subluxation, CTPT, Taping

\section{Introduction}

World health organization defined stroke as the rapid development of localized or global signs of cerebral dysfunction with symptoms lasting more than 24 hours. It can lead to death without any cause apart from vascular origin. ${ }^{1}$ Most of the survivor developed clinical manifestations such as sensorimotor deficits, neurophysiologic disorders, speech disturbances, involuntary movements and other secondary impairments that can impede the patients ADLs. ${ }^{2}$

Most of the stroke patients develop shoulder subluxation in early stage ${ }^{3}$ with occurrence rate of $7-81 \% .{ }^{4}$ Evidence substantiate the fact that onset of flaccid paralysis of the muscles at early stage will be consequential for the stability of shoulder joint. The Gravitational pull on humerus exerts a constant pressure, causes stretch on the joint capsule that further promote inferior shoulder subluxation. ${ }^{5}$ Electromyographic study showed that the supraspinatus and posterior deltoid muscles are the main component to counteract this downward pull. ${ }^{6}$ Hayner $^{7}$ documented that activity of these muscles is absent in early stage of stroke. However, primary cause of shoulder subluxation after stroke is still unknown.

Post stroke shoulder subluxation with pain is reported in 
patients with a rate of incidence as high as $84 \%{ }^{8}$ Though the scarcity of evidence for correlating pain and subluxation still make the occurrence of post stroke shoulder subluxation with pain a debatable matter. ${ }^{8,9}$

Smith et al. $^{5}$ suggested that correct positioning and adequate support of the flaccid shoulder in the initial recovery stage can reduce the prevalence of hemiplegic shoulder subluxation by $5 \%$. Use of arm slings are often suggested by the researchers ${ }^{10}{ }^{11}$ but it has many disadvantages as it encourages a flexion synergy pattern advances to contracture formation, inhibit arm swing and interfere with the body image. 5,12

Researchers suggests that electrical stimulation that can produce a muscle contraction can also be a treatment option of shoulder subluxation. ${ }^{12,13,14}$ In a systemic review Ada and co-authors substantiate the statement by suggesting electrical stimulation used in early stages of stroke have preventive effect on shoulder subluxation, however, it is also documented that once subluxation has occurred, electrical stimulation cannot reduce it effectively. ${ }^{15}$

Various researchers ${ }^{16,17,18,19,20}$ have used strapping method for the shoulder region mainly to provide stability and protection to address hemiplegic shoulder pain and subluxation. Though, the taping method and the taping material used were different, but all of them used adhesive tape. Peters and $\mathrm{Lee}^{20}$ used three piece of tape in the treatment of shoulder subluxation and got effective result. In a pilot study ancliffe ${ }^{16}$ showed that shoulder taping effectively reduce hemiplegic shoulder pain.

California tri-pull taping (CTPT) method when researched in a quasi-experimental study by Hayner ${ }^{7}$ showed significant improvement in subluxation, active range of motion (AROM) and improved activity of daily living skills (ADL's) and also found the tape comfortable.

Taping method used in this study was inspired from the Hayner's ${ }^{7}$ research. Three pieces of rigid tape were used to support the hemiplegic subluxed shoulder. All three pieces were applied from 1.5 inches below the deltoid tuberosity up to mid spine of scapula (posterior), 2 inches above the glenoid fossa (middle) and 1.5 inches above the clavicle (anterior).

The purpose of this pre test and post test study is to lay a foundation for conducting a randomized clinical trial by evaluating the effect of CTPT method on post stroke shoulder subluxation, pain, AROM, and ADLs.

\section{Method}

The study was a pre test post test study across 10 participants ( 7 male, 3 female). Initially 15 participants were recruited for the study among them 3 participants refused to sign consent form and 2 participants refused to take treatment. Baseline measurement was taken to each participant that was amount of shoulder subluxation, pain, AROM, and ADL activities. The intervention phase consists of applying shoulder tape every Monday, Wednesday and Friday for 6 weeks. Post intervention data was taken after 6 weeks to determine whether there is any improvement from the intervention. The study was approved by the ethical review board of MMIPR.

Participants were recruited over a 3 months of time by means of referral from the MM Medical College Mullana, to the outpatient department of MMIPR, Mullana. Inclusion criteria were 1) Acute stroke 2) A minimum of $5 \mathrm{~mm}(0.2$ inch) shoulder subluxation in the involved upper extremity. 3) Pain in the subluxed shoulder. 4) MMSE score $>23.5$ ) Age (35-70 yrs.) and of either sex. Exclusion criteria were 1) MMSE score <23. 2) Other musculoskeletal disorder of the affected upper extremity. 3) History of trauma to the affected upper extremity. 4) Hyper or hypo sensitivity disorders. 5) Any skin allergy. 6) Individual affected from neurological disorder other than stroke. 7) Un-cooperative patients. 8) Individuals with psychosomatic disorder.

\section{Testing Procedure}

Participants were evaluated for acromiohumeral distance (AHD), by using digital Vernier calliper. The measurement was taken in centimetre from the inferior aspect of the acromion to the superior aspect of the humeral head. The patient was in high sitting position such a way that tested upper extremity hanged freely. Paci et $\mathrm{al}^{11}$ stated that calliper method was reliable for shoulder subluxation measurement.

Pain at rest was measured by using a $10 \mathrm{~cm}$ visual analogue scale (VAS). In VAS 1 indicating no pain where 10 indicating extreme pain. Crossleyet $\mathrm{al}^{21}$ reported that VAS is a reliable and valid tool for measurement of pain in patelofemoral pain.

Active flexion (AFLXN) range of motion was assessed using goniometric measurement for shoulder flexion. Active shoulder flexion range was assessed in supine position to avoid trick movement that may happen in sitting position. Hayes et $\mathrm{al}^{22}$ reported that goniometer is a reliable tool for shoulder joint flexion range of motion assessment.

The motor recovery of the upper extremity was assessed by using Fugl-Meyer assessment for upper extremity. The maximum score for upper extremity motor assessment is 66 . Sanford $^{23}$ checked the reliability of the Fugl-Meyer assessment for testing Motor Performance in Patients Following Stroke on 12 patients. The overall reliability was high.

\section{Description of Treatment}

Intervention was given by the lead researcher who was trained for the taping techniques.

Prior to applying tape, participants were instructed shave the hair around the shoulder joint. Two types of tape was used, a self-adhesive 1.5" cotton undercover tape (VPK, Chennai) and a 1" rigid strapping tape (VPK, Chennai). To approximate the humeral head into the glenoid cavity participants were instructed to place their affected arm on a wooden table. Taping method contained a cotton undercover pre tape and then the rigid post tape on it. Three 
piece of tape was used, First piece (middle) of tape was applied from 1.5 inches below the deltoid tuberosity up to 2 inches above the glenoid cavity. Second piece (posterior) was applied from 1.5 inches below the deltoid tuberosity upto the 1.5 inches above the mid spine of scapula. Third piece (anterior) was applied from the 1.5 inches below the deltoid tuberosity upto the 1.5 inches above the clavicle. New tape applied every Monday, Wednesday, and Friday till 6 weeks. (figure: 1,2,3,4).

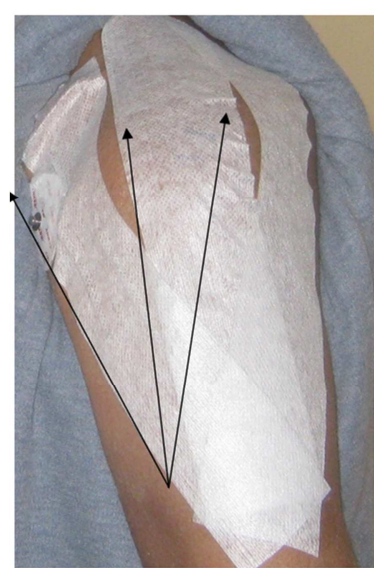

Figure 1

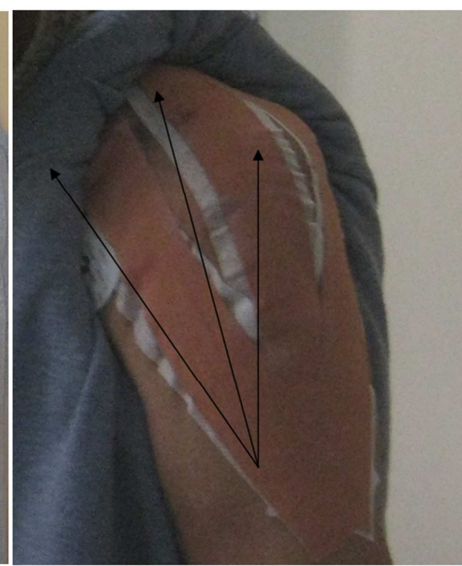

Figure 2

Figure 1., Figure 2. Application of pretape Application of post tape

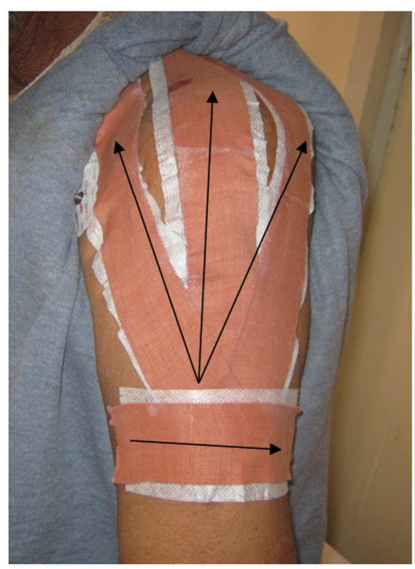

Figure 3

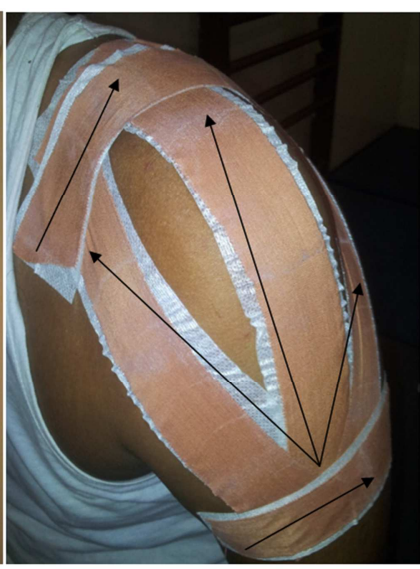

Figure 4
All the participants were received standardized conventional neurorehabilitation programme. The conventional neurorehabilitation treatment which includes active and passive range of motion, neuromuscular reeducation, tone normalization, weight bearing exercise of upper extremity, functional reach, grasp, hold and release activities and ADL activities. Every participant received conventional neuro-rehabilitation for 45 minutes a day, 5 days a week for six weeks.

\section{Statistical Analysis}

Pre intervention and post intervention data was collected by a completely blind observer, who was having Master in physiotherapy degree with 2 years of clinical experience.

Data analysis was done by using SPSS version 22.0 software with $95 \%$ confidence interval. The level of significance was set at 0.05 .

Paired t-test was used to compare pre and post mean values of all variables (VC, VAS, AFLXN, and FUG).

\section{Results}

Ten participants were enrolled in the study, 7 men and 3 women. Eachone of them was a diagnosed case of stroke with inferior shoulder subluxation. Onset of stroke was, for 4 participants were 1 week, 2 participants were between 2-4 week, 4 participants were between 6-7 week post stroke.

After the treatment all the participants showed a significant improvement $(\mathrm{p}=0.00)$ in all clinical parameters. (Table 1)

Amount of reduction of shoulder subluxation was significant, mean difference of pre-test and post-test score was $0.773(\mathrm{p}=0.000)$. Mean difference of pre-test and posttest score for reduction of pain was $2.30(\mathrm{p}=0.000)$ which is statistically significant. Improvement of active shoulder flexion range was also found to be statistically significant. Mean difference of pre-test and post-test score was 12.20 $(\mathrm{p}=0.000)$. Activity of daily living was also ameliorate significantly. Mean difference of pre-test and post-test score was $16.20(\mathrm{p}=0.000)$.

Figure 3., Figure 4. California tri-pull taping method

Table 1. Pretest and postest scores of the participants.

\begin{tabular}{|c|c|c|c|c|c|c|}
\hline Outcome measure & $\begin{array}{l}\text { Pre-test Mean } \pm \text { SD } \\
(95 \% \text { CI })\end{array}$ & $\begin{array}{l}\text { Post-tests Mean } \pm \text { SD } \\
(95 \% \text { CI) }\end{array}$ & $\begin{array}{l}\text { Mean difference } \\
\text { (SEM) }\end{array}$ & Test score & p value & $\begin{array}{l}\text { Effect size } \\
\text { (Cohen's d) }\end{array}$ \\
\hline AHD & $\begin{array}{l}2.72 \pm 0.820 \\
(2.21-3.23)\end{array}$ & $\begin{array}{l}1.94 \pm 0.706 \\
(1.5-2.38)\end{array}$ & $\begin{array}{l}0.773 \\
(0.134)\end{array}$ & 5.728 & 0.000 & 1.01 \\
\hline VAS & $\begin{array}{l}7.40 \pm 1.506 \\
(6.47-8.33)\end{array}$ & $\begin{array}{l}5.10 \pm 1.792 \\
(3.99-6.21)\end{array}$ & $\begin{array}{l}2.30 \\
(0.367)\end{array}$ & 6.273 & 0.000 & 1.38 \\
\hline AFLXN & $\begin{array}{l}12.10 \pm 2.079 \\
(10.81-13.39)\end{array}$ & $\begin{array}{l}24.30 \pm 7.288 \\
(19.78-28.82)\end{array}$ & $\begin{array}{l}12.20 \\
(1.867)\end{array}$ & 6.536 & 0.000 & 2.27 \\
\hline FMA & $\begin{array}{l}11.10 \pm 1.370 \\
(10.25-11.95)\end{array}$ & $\begin{array}{l}16.20 \pm 2.486 \\
(14.66-17.74)\end{array}$ & $\begin{array}{l}5.10 \\
(0.623)\end{array}$ & 8.190 & 0.000 & 2.54 \\
\hline
\end{tabular}

AHD: Acromio humeral distance, VAS: Visual analogue scale, AFLXN: Active flexion, FMA: Fugel-Mayer assessment of upper extremity. CI: Confidence interval. 


\section{Discussion}

The major findings of the study was that California tripull taping (CTPT) method was effective in reducing post stroke shoulder subluxation, pain and improving active flexion range (AFLXN) and upper limb function. Statistical analysis reveals that participants showed significant improvement in all the clinical parameters.

Participants exhibit significant reduction in Accromio humeral distance (AHD) on digital Vernier caliper. The mean reduction of the AHD was $0.77 \mathrm{~cm}$ with an effect size of 1.01. This finding is corroborate by previous study of Hayner. $^{7}$ that calculated $0.78 \mathrm{~cm}$ of mean AHD reduction.

The main effect of CTPT method in reduction of AHD was that, it will provide a proprioceptive feedback through the mechanoceptors in the skin, muscle, fascia, tendon and articular structures. This promotes position sense, kinesthesia and force detection. It also keep the underactive muscles in a shortened position which improve actin myosin cross bridge formation and there was a shift of length tension curve towards left and force development was increases in the underactive muscle groups. ${ }^{24}$

One important finding of the study was significant reduction of post stroke shoulder pain on VAS scale. In the present study the mean pain reduction was $2.30 \mathrm{~cm}$ on VAS with an effect size of 1.38 , which was nearer to the MCID value of VAS $(3 \mathrm{~cm}) .{ }^{25}$ In a previous study Pandian et $\mathrm{al}^{26}$ showed $1.9 \mathrm{~cm}$ of mean pain score changes in between groups on VAS, but the study was conducted with hemiplegic shoulder pain participants not with shoulder subluxation patients.

The possible effect of CTPT method on pain reduction was, it provide protection of the joint from further trauma during active and passive movement, ambulation and ADLs, as it reaproximate the head of the humerus into the glenoid fossa by assisting the rotator cuff muscles. ${ }^{7}$

In the present study AFLXN was improved significantly, the mean improvement of AFLXN was 12.2 degree with an effect size of 2.27, which was also reached the MCID value of AFLXN that is 11 degree. ${ }^{27}$ AFLXN was improved because CTPT method helps to improve the force generation and motor control of the musculature surrounding shoulder joint. When stroke survivors gain some movement in there affected extremity they were incorporated the affected arm more actively in the daily living. When this happens, active range increases. ${ }^{28}$

Upper limb motor recovery was improved significantly on Fugl-Meyer scale, the mean improvement was 5.1 with an effect size of 2.54 . In a previous study, Hyner, ${ }^{7}$ also showed that CTPT method improved the functional abilities of the participants. Mean improvement of functional ability was 1.07 on Katz index of daily living.

Major limitation of the study was participants could not be follow up after the study because of limited time frame so the long term effect of CTPT method was unknown. Radiographic measurement of the shoulder subluxation was not taken so exact amount of improvement of shoulder subluxation cannot be interpreted. As there was no control group we cannot conclude about the effectiveness of CTPT method.

\section{Conclusion}

Despite of various limitations our study showed that CTPT method is a promising treatment method for post stroke shoulder subluxation. Randomized clinical trial is needed with large sample size and radiographic measurement to establish the effectiveness of CTPT method.

\section{List of Abreviations:}

ADL: Activity of daily living

AFLXN: Active flexion range

AHD:Accromio humeral distance

CTPT: California tri-pull taping

FUG: Fugl- Meyer assessment of upper extremity.

MCID: Minimal clinical important difference

ROM: Range of motion.

SD: Standard deviation.

VAS: Visual analogue scale.

VC: Digital Vernier calliper

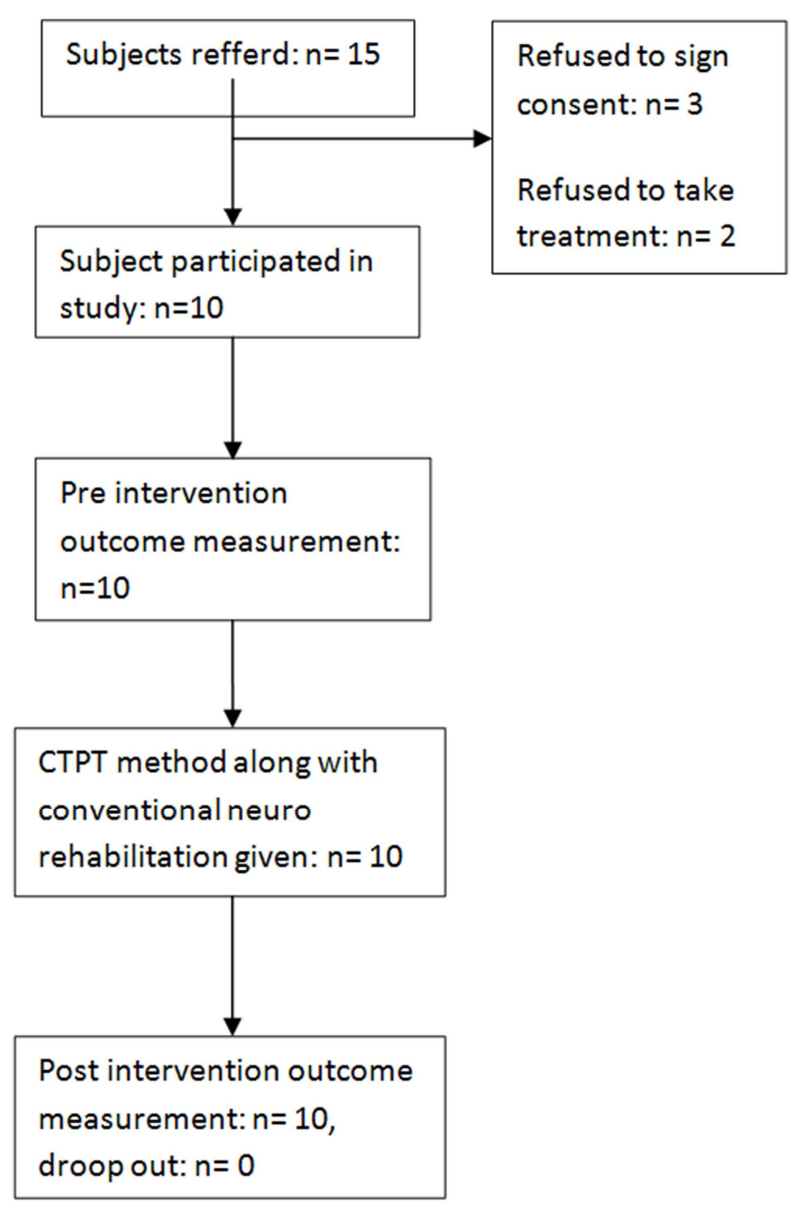

Participants flow chart 


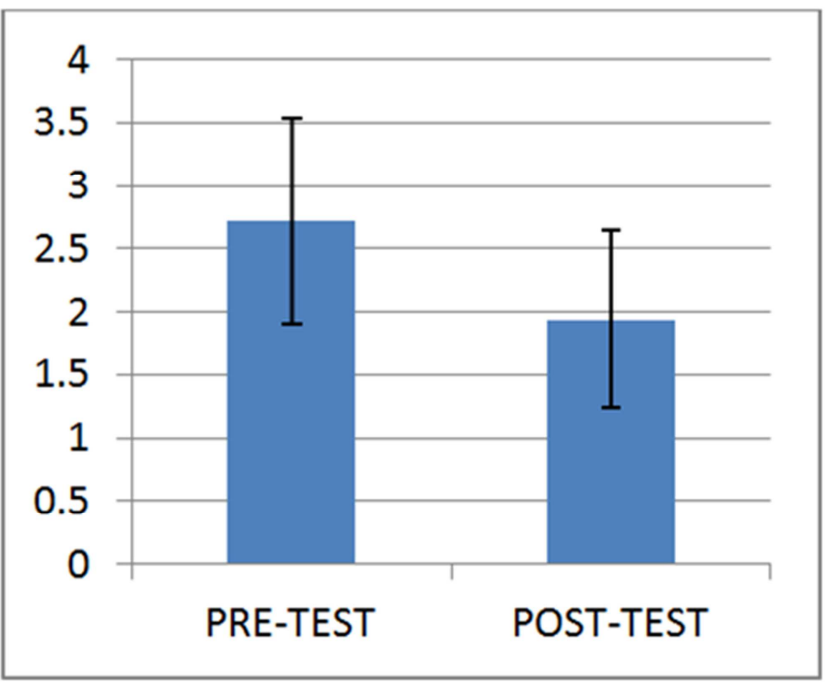

Graph 1. Comparison of pre-test and post-test scores of $A H D$.

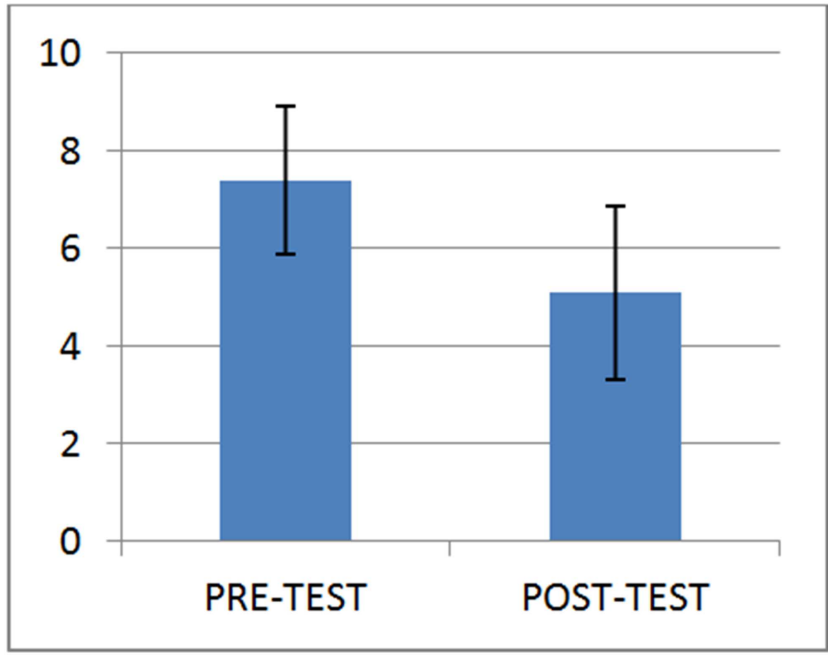

Graph 2. Comparison of pre-test and post testscores of VAS.

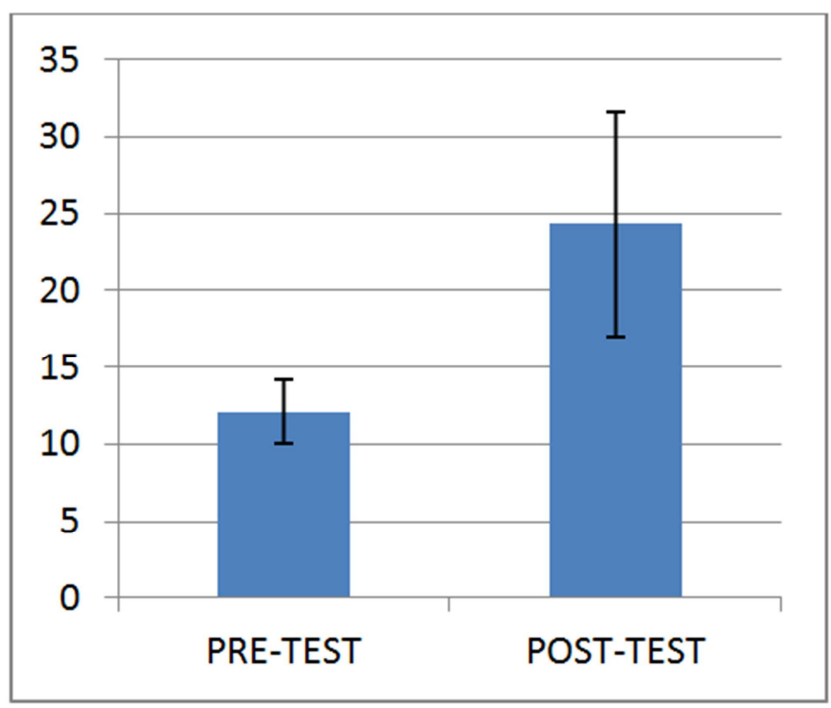

Graph 3. Comparison of pre-test and post-testScores of VAS.

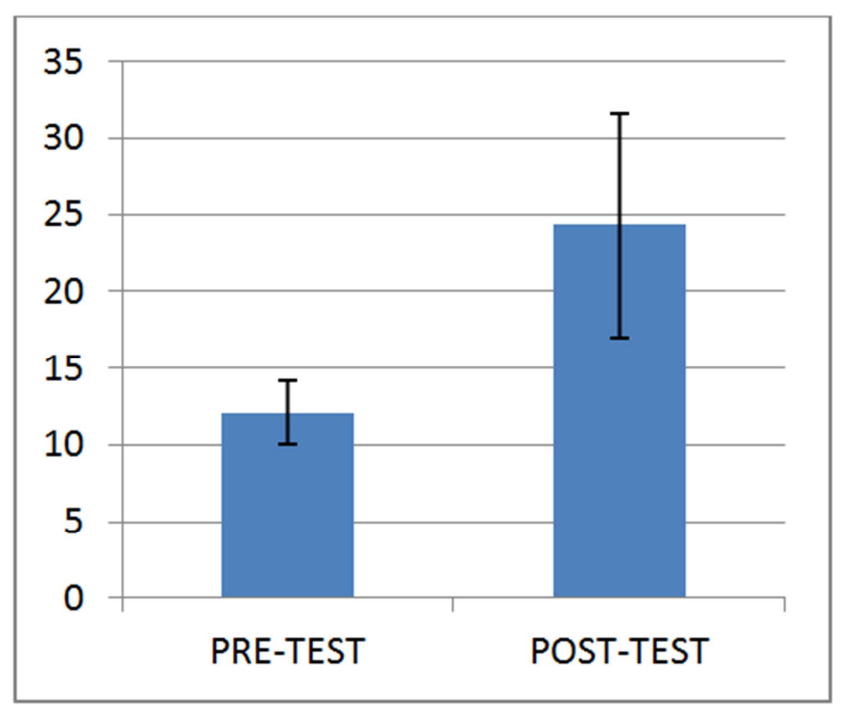

Graph 4. Comparison of pre-test and post-testscores of FMA.

\section{References}

[1] Evelyne AD, Mamadou Z, Dine MW, Leonard KK, C Ehounoud Y. Current data on the profile of patients hospitalized for stroke in a neurology department in west Africa: Abidjan (Ivory Coast). Am J Psychiatr Neurosci. 2015; 3: $14-21$.

[2] Assogba $\mathrm{K}$ et al. Arterial Territories Affected in the Thalamic Stroke: A Prospective Study Among Inpatients in Lome University Hospital, Togo. Am J Psychiatr Neurosci. 2015; 3: $9-13$.

[3] Najenson T, Yacubovich E, Pikielni SS. Rotator cuff injury in shoulder joints of hemiplegic patients. Scand J Rehabil Med.1971; 3: 131-37.

[4] Najeson $\mathrm{T}$ et al. Mal-alignment of the glenohumeral joint following hemiplegia. Ann Phys Med. 1965; 8: 96-99.

[5] Smith RJ, Cruikshank JG, Dunbar S, Akthar AJ. Malalignment of the shoulder after Stroke. Br Med J.1982; 284: $1224-26$.

[6] Basmajian, JV, \& Bazant FJ. Factors preventing downward dislocation of the adducted shoulder joint: An electromyographic and morphological study. J Bone Joint Surg Am. 1959; 41-A: 1182-1186.

[7] Hayner KA. Effectiveness of the California tri-pull taping method for shoulder subluxation post stroke: A single subject ABA design. Am J Occup Ther. 2012; 66: 727-36.

[8] Zorowitz DR, Hughes MB, Idank D, Ikai T, Johnston MV. Shoulder Pain and Subluxation after Stroke: Correlation or Coincidence? Am J Occup Ther. 1996; 50: 194-01.

[9] Suethanapornkul S, Kuptniratsaikul SP, Kuptniratsaikul V, Uthensut V. Post Stroke Shoulder Subluxation and Shoulder Pain: A Cohort Multicentre Study. J Med Assoc Thai. 2008; 91(12):1885-93.

[10] Ada L, Foongchomcheay A, Canning CG. Supportive devices for preventing and treating subluxation of the shoulder after stroke. Stroke. 2005; 36: 1818-19. 
[11] Paci M, Nannetti L, Rinaldi AL. Glenohumeral subluxation in hemiplegia: An overview. J Rehabil Res Dev. 2005; 42(4):557-68.

[12] Zorowitz RD, Idank D, lkai T, Hughes MB, Johnston MV. Shoulder Subluxation after Stroke: A Comparison of Four Supports. Arch Phys Med Rehabil. 1995; 76: 763-71.

[13] Baker LL, Parker K. Neuromuscular electrical stimulation surrounding the shoulder. Phys Ther. 1986; 66: 1930-37.

[14] Kobayashi H, Onishi H, Ihashi K, Yagi R, Handa Y. Reduction in subluxation and improved muscle function of the hemiplegic shoulder joint after therapeutic electrical stimulation. J Electromyogr Kinesiol.1999; 9: 327-336.

[15] Ada L, Foongchomcheay A. Efficacy of electrical stimulation in preventing or reducing subluxation of the shoulder after stroke: A meta-analysis. Aust J Phys Ther. 2002; 48: 257-67.

[16] Ancliffo J. Strapping the shoulder in patients following a cerebrovascular accident (CVA): A pilot study. Aust J Phys Ther. 1992; 38(1):37-41.

[17] Hanger HC, Whitewood P, Brown G, Ball M, Harper J, Cox R, Sainsbury R. A randomised control trial of strapping to prevent post-stroke shoulder pain. Clin Rehabil. 2000; 14(4):370-80.

[18] Morin L, Bravo G. Strapping the hemiplegic shoulder: A radiographic evaluation of its efficacy to reduce subluxation. Phys Can.1997; 49: 103-108.

[19] Morin GE, Tiberio D, Austin G. The effect of upper trapezius taping on electromyographic activity in the upper and middle trapezius region. J Sports Rehabil. 1997; 6: 309-318.
[20] Peters SB, Lee GP. Functional impact of shoulder taping in the hemiplegic upper extremity. Occup Ther Health Care 2003; 17: 35-46.

[21] Crossley KM, Bennell KL, Cowan SM, Green S. Analysis of outcome measures for persons with patellofemoral pain: Which are reliable and valid? Arch Phys Med Rehabil. 2004; 85: 815-22.

[22] Hayes K, Walton JR, Szomor ZR, Murrell GA. Reliability of five methods for assessing shoulder range of motion. Aust $\mathrm{J}$ Physther. 2001; 47: 289-94.

[23] Sanford J, Moreland J. Reliability of the Fugl-Meyer Assessment for Testing Motor Performance in Patients Following Stroke. Phys Ther. 1993;73:447-54

[24] Kneeshaw D. Shoulder taping in the clinical setting. J Bodyw Mov Ther. 2001; 6(1):2-8

[25] Lee SJ, Hobden E, Stiell IG, Wells GA. Clinically important change in the Visual Analog scale after adequate pain control. Acad Emerg Med. 2003; 10 (10):1128-30.

[26] Pndian JD et al. Shoulder taping reduces injury and pain in stroke patients: randomized controlled trial. Neurology. 2013; 80: 528-532.

[27] Susan W, Charlene L, Lauren B. Evaluating change in clinical status: Relibility and measures of agreement for the assessment of glenohumeral range of motion. N Am J Sports Phys Ther. 2010; 5: 98-110.

[28] Zorowitz RD. Recovery patterns of shoulder subluxation after stroke: A six-month follow-up study. Top Stroke Rehabil. 2001; 8(2):1-9. 